JOURNAL OF COMPUTATIONAL BIOLOGY

Volume 16, Number 2, 2009

(C) Mary Ann Liebert, Inc.

Pp. 169-180

DOI: $10.1089 / \mathrm{cmb} .2008 .04 T T$

\title{
Bottlenecks and Hubs in Inferred Networks Are Important for Virulence in Salmonella typhimurium
}

\author{
JASON E. McDERMOTT, ${ }^{1}$ RONALD C. TAYLOR, ${ }^{1}$ HYUNJIN YOON, ${ }^{2}$ \\ and FRED HEFFRON ${ }^{2}$
}

\begin{abstract}
Recent advances in experimental methods have provided sufficient data to consider systems as large networks of interconnected components. High-throughput determination of proteinprotein interaction networks has led to the observation that topological bottlenecks, proteins defined by high centrality in the network, are enriched in proteins with systems-level phenotypes such as essentiality. Global transcriptional profiling by microarray analysis has been used extensively to characterize systems, for example, examining cellular response to environmental conditions and effects of genetic mutations. These transcriptomic datasets have been used to infer regulatory and functional relationship networks based on coregulation. We use the context likelihood of relatedness (CLR) method to infer networks from two datasets gathered from the pathogen Salmonella typhimurium: one under a range of environmental culture conditions and the other from deletions of 15 regulators found to be essential in virulence. Bottleneck and hub genes were identified from these inferred networks, and we show for the first time that these genes are significantly more likely to be essential for virulence than their non-bottleneck or non-hub counterparts. Networks generated using simple similarity metrics (correlation and mutual information) did not display this behavior. Overall, this study demonstrates that topology of networks inferred from global transcriptional profiles provides information about the systems-level roles of bottleneck genes. Analysis of the differences between the two CLR-derived networks suggests that the bottleneck nodes are either mediators of transitions between system states or sentinels that reflect the dynamics of these transitions.
\end{abstract}

Key words: bottlenecks, network inference, Salmonella typhimurium, virulence.

\footnotetext{
${ }^{1}$ Computational Biology \& Bioinformatics Group, Pacific Northwest National Laboratory (U.S. Department of Energy), Richland, Washington.

${ }^{2}$ Department of Molecular Microbiology and Immunology, Oregon Health \& Science University, Portland, Oregon.
} 


\section{INTRODUCTION}

$\mathbf{T}$

HE ELUCIDATION OF BIOLOGICAL NETWORKS of different types has become tractable largely because of the increase in data provided by high-throughput methods for experimental measurement. Computational methods designed to infer subtle relationships between genes and/or proteins have also been developed to mine global transcriptional data generated by large numbers of microarray experiments. These network inference methods have been used to determine detailed genetic regulatory networks (Faith et al., 2007) as well as general functional association networks which link, for example, co-regulated groups of genes (Horvath and Dong, 2008; Reiss et al., 2006). The promise of such global networks is that they can be harnessed to provide useful biological insight into the systems or the components that might not have been apparent by investigating individual components in isolation.

Protein interaction, transcriptional regulatory, and metabolic networks are different ways of looking at the underlying "master" network of connected cellular processes at multiple levels (mRNA, protein, metabolism) in the cell. Each of these network views have particular structure dictated by evolutionary processes. In the most fundamental sense, they must function to ensure the survival of the organism with which they are associated. The local topology of these networks has been used to predict various systems-level phenotypes. One study has reported that hubs, highly connected, are more likely to be lethal if removed by mutagenesis (Jeong et al., 2001). Other studies have found that hubs evolve more slowly (Fraser et al., 2002) and are enriched in proteins involved in aging (Ferrarini et al., 2005; Pletcher, 2004; Promislow, 2004) or recovery from DNA damage (Said et al., 2004).

Global network topology measures, such as betweeness centrality, are also well correlated with essentiality and evolutionary rates (Yu et al., 2007). Betweeness is calculated as the percentage of times that a protein appears in the shortest path between all pairs of proteins in the network. Proteins with a high level of betweeness, called bottlenecks, can be thought of as controlling information flow in the network, similar to bridges linking two parts of a city. The importance of bottlenecks has been demonstrated in a variety of experimentally determined interaction networks by showing that these proteins are significantly more likely to be essential when deleted in yeast (Yu et al., 2007); 34\% of bottleneck proteins were found to be essential versus $18 \%$ of non-bottleneck proteins. Recently, a number of approaches analyzed bottlenecks in human protein-protein interaction networks. These studies have identified genes involved in neurodegenerative diseases (Goni et al., 2008), and characterized properties of successful drug targets (Yao and Rzhetsky, 2008) and targets of different pathogens (Dyer et al., 2008). Bottleneck nodes in other types of networks, for example those derived from mining literature, have also been used to identify disease-associated genes (Ozgur et al., 2008).

The network topology studies described here have analyzed experimentally determined protein-protein interaction networks, genetic regulatory networks, and metabolic networks: no correlation of bottlenecks from inferred networks with systems-level phenotypes has been reported. Generally, experimental determination of interaction networks in an organism is expensive and can be significantly error-prone depending on the experimental method used (von Mering et al., 2002). Global transcriptomic data is much easier to obtain, and can be used to infer networks of regulatory and functional associations between genes. The utility of analyzing the topology of inferred networks from transcriptomic data from the model pathogen Salmonella typhimurium is the focus of the current study.

Salmonella typhimurium (formally Salmonella enterica serovar Typhimurium) is the causative agent of gastroenteritis in humans and a lethal systemic infection in susceptible BALB/c mice (Jones and Falkow, 1996). A major challenge in bacterial pathogenesis is to unravel how multiple environmental cues are processed and integrated into a logical and coordinated cellular response. Successful response to these environmental requirements is accomplished through an elaborate regulatory program which controls the expression patterns of a large number of genes involved in virulence (Milo et al., 2002, 2004; Yoon et al., 2009).

Identification of genes and their products that are essential for this virulence program is an area of considerable experimental investigation (Fields et al., 1986; Galan and Collmer, 1999; Geddes et al., 2005; Worley and Heffron, 2000). Virulence proteins and those essential for virulence represent both the arsenal the bacteria uses to establish a productive infection as well as the Achilles heel of the pathogen, which can be targeted to therapeutically treat such infections. In S. typhimurium, there have been several studies using high-throughput experimental methods for identification of such virulence proteins (Chan et al., 2005; Lawley et al., 2006; Morgan et al., 2004; Shah et al., 2005), and we use these lists of essential genes in this study. 
We report that network topology, specifically determination of genes that are hubs and bottlenecks, can be usefully applied to networks inferred from sets of global transcriptional profiles for S. typhimurium using the context likelihood of relatedness (CLR) algorithm. We inferred networks from two independent transcriptomic datasets: one a study of 32 different culture conditions (GSE2456) and the other a study of genetic mutations in transcriptional regulators involved in virulence (15regulators). We report that genes identified as hubs or bottlenecks are significantly more likely to be essential for virulence than non-bottlenecks in the networks inferred using CLR. Furthermore, the subset of bottlenecks that are also hubs are even more likely to be essential than non-hubs and non-bottlenecks. We also show that a set of bottleneck genes shared between the two inferred networks are further enriched in virulence-essential genes. These relationships are not observed in networks constructed from the GSE2456 dataset using mutual information alone or simple Pearson correlation between expression profiles, thus indicating that the statistical filtering of relationships by the CLR algorithm produces more biologically useful networks. Functional analysis of these bottleneck nodes and significantly enriched functional categories show that these nodes are preferentially involved in processes that reflect the underlying experimental design, for instance, utilization of different carbon sources. These results suggest that bottleneck nodes from inferred networks may represent either mediators of systems transitions between states (e.g., use of different carbon sources) or sentinels of these transitions.

\section{METHODS}

\subsection{Datasets used}

To investigate the role of bottleneck genes in networks inferred from transcriptional profiles, we used two datasets from experimental studies of $S$. typhimurium. The first dataset used is from a series of $S$. typhimurium microarray experiments generated in Dr. Michael McClelland's laboratory at Scripps Research Institute and available from the National Institutes of Health (NIH) GEO database (G. Yun et al., unpublished data; GEO accession number GSE2456). This dataset includes microarrays from 32 different growth conditions, with three biological replicates each. These conditions represent a basic growth curve experiment, in which gene response during maximal growth on a variety of media was measured. The growth conditions include several different carbon sources, anaerobic conditions, $\mathrm{pH}$ variations, and addition of chelators and several other biologically active compounds. A number of conditions known to stimulate virulence response in $S$. typhimurium are represented, including low $\mathrm{pH}$ and low magnesium. The final dataset included 26 different conditions for 4696 genes after filtering to exclude several conditions tested in a different Salmonella strain. We refer to the network generated from this dataset as the "GSE2456 network" in this study.

Recently, we analyzed the effects of deleting 15 transcriptional regulators essential for S. typhimuirum virulence ( $s p v R$, fruR, himD, phoP/Q, ssrA/B, slyA, hnr, rpoE, smpB, csrA, rpoS, STM3120, crp, ompR/envZ, and $h f q$ ) in mice by global transcriptional profiling (Yoon et al., 2009) also performed in Dr. McClelland's lab. Three biological replicates for each mutant were grown under four different conditions; exponential growth in rich media (Luria-Bertani [LB]), stationary phase growth in rich media, and two culture variants of a low-pH, low $\mathrm{Mg}^{2+}$ minimal media $(\mathrm{MgM})$ designed to mimic the intracellular environment of the macrophage and shown to induce the virulence program in S. typhimurium (Adkins et al., 2006). The biological replicates were pooled, mRNA was isolated, converted to cDNA, and applied to a pan-Salmonella microarray (Porwollik et al., 2003). This resulted in 63 individual datasets representing different regulator mutant/culture condition combinations, including duplicate copies of the parental strain used as a control. One array was omitted from a specific growth condition (crp: one MgM media condition), because it did not grow in this minimal media. We refer to the network generated from this dataset as the " 15 regulator network" in this study.

Homology relationships between S. typhimurium and Escherichia coli proteins are from the Bioverse database (Guerquin et al., 2009; McDermott et al., 2005). These relationships were generated using PSIBLAST (Altschul et al., 1997) with three iterations and calculating the sequence identity of resulting matches (below an evalue threshold of 1.0) relative to the entire query sequence. 


\subsection{Network inference}

We used the previously developed CLR algorithm (Faith et al., 2007), which has been included in the Software Environment for BIological Network Inference (SEBINI) (Taylor et al., 2006) framework, using 10 bins and a spline degree of three as input parameters. For both datasets, all genes were considered so the inferred relationships between genes represent all functional associations rather than just the transcriptional regulatory interactions as in Faith et al. (2007), though these should be present as well. For selection of the highest-scoring interactions, we first converted the CLR scores to zscores. We chose the zscore thresholds for the networks from the analysis shown in Figure 1A, based on the best enrichment of the VirEss measure in bottlenecks relative to non-bottlenecks. To maintain a fair comparison between the two networks, we chose the threshold for the 15regulator CLR network to keep a similar number of genes in that network. The chosen threshold was 7.6 for the GSE2456 network giving a network with 748 genes, and 5.2 for the 15regulator network giving a network of 692 genes.

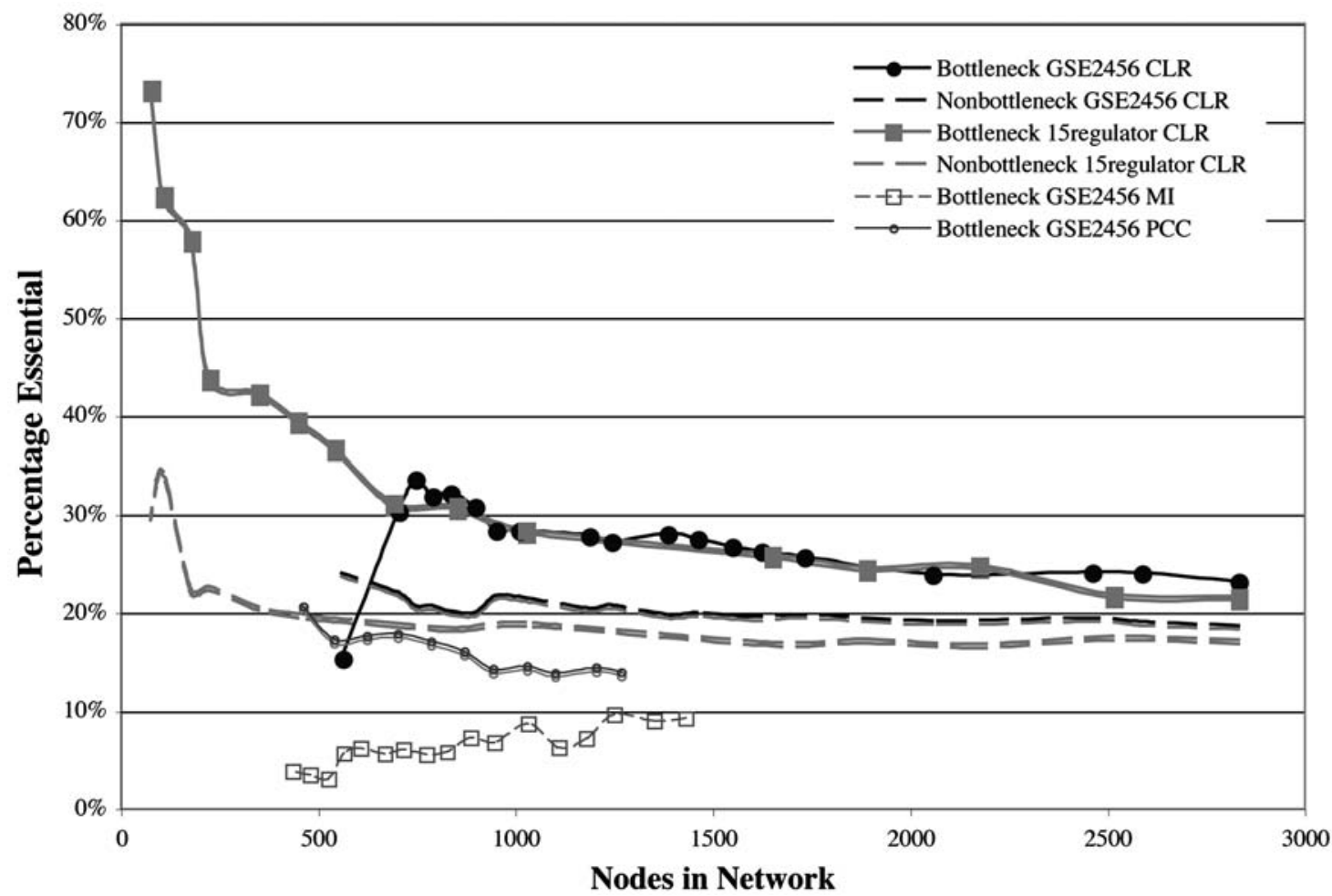

(A)

FIG. 1. Bottleneck nodes from inferred networks are more likely to be essential in virulence. (A) Networks were inferred from the GSE2456 (closed circles) or 15regulator (closed squares) dataset using the context likelihood of relatedness (CLR) algorithm, mutual information (MI; open squares), or Pearson correlation (PCC; open circles) as described in the text. We varied the thresholds used for generation of the networks which are reflected in the size of the network ( $X$ axis) since more conservative thresholds generate smaller networks. The mean virulence essentiality (VirEss) was calculated for the bottleneck nodes (solid lines) and non-bottleneck nodes (dashed lines) and expressed as the percentage of genes in the group which are essential for virulence under at least one of the genetic screens examined (see text). Only those data points which show a statistically significant difference $(p$-value $<0.05)$ are shown for the two CLR networks. Similar results were observed by examining hub versus nonhub genes (data not shown). (B) Networks were generated using thresholds chosen from panel A (see text) and the percentage of essential genes in different categories, bottlenecks, nonbottlenecks, hub-bottlenecks, nonhub-bottlenecks, and hub-nonbottlenecks was calculated. The observed differences for the relationships from the CLR networks are significant. These results show that hub-bottlenecks are the most enriched in essential genes and that nonhub-bottlenecks and hub-nonbottlenecks are equivalently enriched in the 15regulator network indicating hubs and bottlenecks are important in different ways.

(continued) 


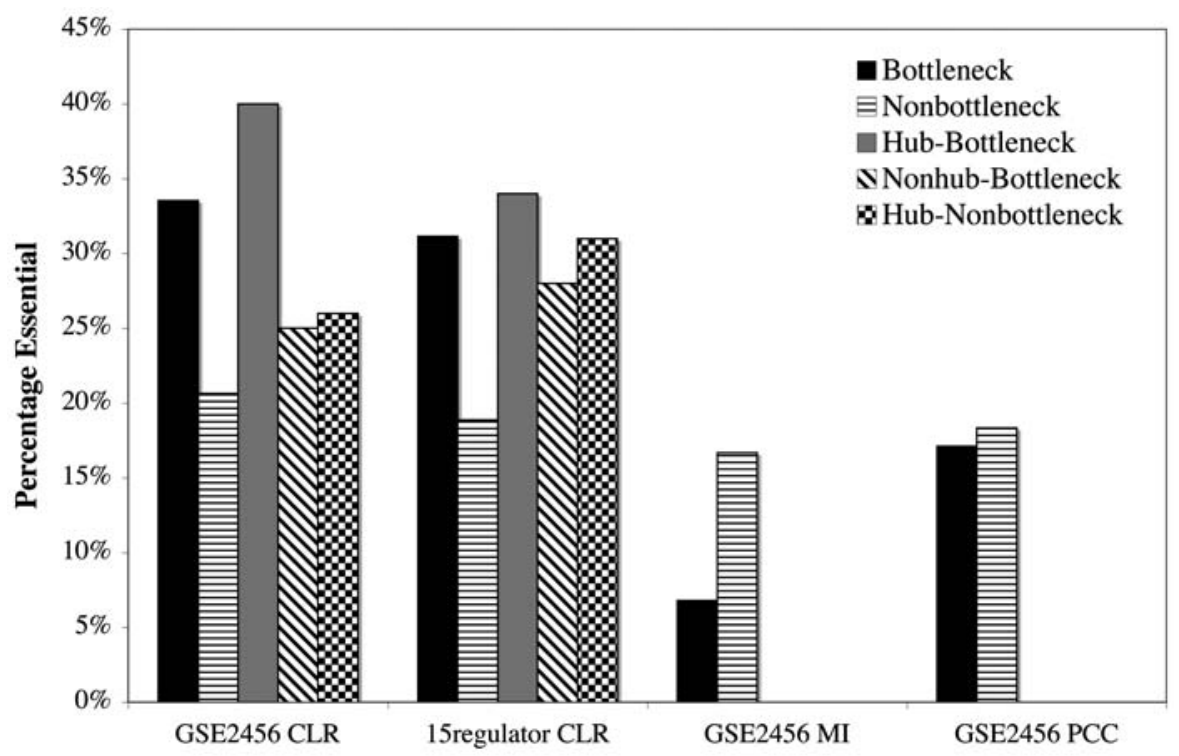

(B)

FIG. 1. (Continued).

We also analyzed the GSE2456 dataset using the raw mutual information values output by the CLR algorithm (i.e., before statistical filtering of relationships) and Pearson correlation coefficients (PCC) between gene expression profiles. We chose thresholds for further comparison to approximately match the size of the CLR networks described above. These networks are referred to as "GSE2456 MI" and "GSE2456 PCC," respectively.

\subsection{Network topology}

Network topology was calculated using the NetworkX module (http://networkx.lanl.gov) for Python as implemented in the Bioverse pipeline (Guerquin et al., 2009; McDermott et al., 2005). We used the brandes_betweenness_centrality function in NetworkX, which is the number of times a node is included in the shortest paths between all pairs of genes in the network normalized by the total number of paths.

\subsection{Statistical analysis}

We calculated the statistical significance of enrichment of various features in bottlenecks versus nonbottleneck genes from the networks examined. For binary categorical data (the VirEss measure and enrichment of KEGG functional pathways), we used a chi-square test to assess significance, taking a chi-square statistic of 3.84 or above, which corresponds to a $p$-value of less than 0.05 , as statistically significant. For the other features (\%GC, E. coli homology, macrophage, and MgM expression), we used the Student's $t$-test, again considering a $p$-value of 0.05 as statistically significant. Finally, we used a multinomial test to assess the significance of the VirSum metric, again considering a $p$-value of less than 0.05 as significant.

\section{RESULTS AND DISCUSSION}

To examine the importance of genes in S. typhimurium, we compiled a list of genes found to be essential in virulence by genetic negative selection screens (Chan et al., 2005; Lawley et al., 2006; Morgan et al., 2004; Shah et al., 2005). These methods, signature-tagged mutagenesis (STM) and in vivo expression technology (IVET), both allow identification of large numbers of virulence-essential genes by constructing libraries of genetic mutations in a population of bacteria (Angelichio and Camilli, 2002). This library is 
used to infect a host or cell culture, and the surviving bacteria are isolated at some point after infection. Genes containing insertions or deletions that are not observed in the post-infection bacterial population are indicated to be essential for the process of infection in that host or cell type. The five lists from these studies show a low degree of overlap with each other, a property previously observed in negative selection screens for Yersinia pestis virulence (Darwin, 2005). The low concordance is likely because each screen was performed using slightly different methods, in different cell types and/or host organisms, and in some cases with different transposons. For this reason, we calculated two measures of virulence essentiality that summarize the results from all five lists. The first, VirEss, is 1.0 if a gene was found to be essential by any of the studies; otherwise, it is assigned a 0 . The second, VirSum, is the sum of all occurrences of a gene in each of the lists. This metric discriminates genes which are essential under a narrow range of conditions from those that are essential in a broader range of conditions, albeit differences in the transposon employed may have been responsible for different insertion specificity.

We first used the CLR algorithm to infer relationships between gene expression profiles from each dataset, the GSE2456 environmental conditions study and the 15regulator mutant study. We used a range of threshold values to construct networks of varying sizes and examined the enrichment of genes essential in virulence (VirEss) in bottleneck nodes for each threshold (Fig. 1A). Network hubs and bottlenecks were identified by ranking the genes in the network according to their degree (the number of relationships the gene has in the network) or their Brandes betweeness centrality (bottlenecks), and considering the top 20\% to be hubs or bottlenecks as in Yu et al. (2007). Betweeness is the number of times a node is included in the shortest paths between all pairs of nodes in the network.

The hypothesis that the distribution of essential genes in the sets of bottleneck and non-bottleneck nodes are from the same distribution (the null hypothesis) was tested using the chi-square test; only those comparisons with a $p$-value less than 0.05 were considered to be significant. The comparison between bottlenecks and non-bottlenecks in both the GSE2456 and 15regulator networks over a range of thresholds (Fig. 1A) shows that bottlenecks in both the CLR-derived networks are significantly more likely to be essential in virulence than non-bottlenecks. Thresholds for further analysis were chosen by selecting the maximum enrichment in the GSE2456 CLR network (zscore of 7.6) and then selecting the zscore which generated a network of similar size (and similar enrichment) in the 15regulator CLR network (zscore 5.2). We found the same relationship held between hub and non-hub nodes in the network; in the GSE2456 CLR network ( $z$ score 7.6 ), $33.8 \%$ of hubs were found to be essential, whereas $20 \%$ of the non-hubs were essential in virulence. This is particularly interesting given that $55-70 \%$ of the bottlenecks were found to be hubs. Contrary to the observation that degree and betweeness are highly correlated in protein-protein interaction networks (correlation 0.49) and regulatory networks (0.67), we found a correlation of 0.26 (GSE2456) and 0.40 (15regulator) in inferred networks. This indicates that bottleneck and hub genes in these networks may play very different roles.

Figure 1B shows the enrichment of the VirEss measure in bottleneck categories from both networks. As previously observed from topological analysis of protein-protein interaction networks (see Fig. 2 of Yu

Table 1. Characteristics of Bottleneck Nodes IN INFERRED NETWORKS

\begin{tabular}{lccccc}
\hline & \multicolumn{2}{c}{ 15regulator CLR } & & \multicolumn{2}{c}{ GSE2456 CLR } \\
\cline { 2 - 3 } \cline { 6 - 7 } & \multicolumn{1}{c}{$B N$} & $N B N$ & & $B N$ & $N B N$ \\
\hline Number & 138 & 554 & & 156 & 623 \\
G+C & $\mathbf{5 0 \%}$ & $\mathbf{5 2 \%}$ & & $51 \%$ & $52 \%$ \\
Escherichia coli & $\mathbf{5 9 \%}$ & $\mathbf{6 6 \%}$ & & $69 \%$ & $69 \%$ \\
Macrophage & & & & & \\
$\quad$ 8 h post-infection & $\mathbf{1 . 4}$ & $\mathbf{1 . 9}$ & & 1.3 & 1.5 \\
$\quad$ 12 h post-infection & 2.4 & 1.8 & & $\mathbf{2 . 7}$ & $\mathbf{1 . 5}$ \\
MgM & $\mathbf{3 . 8}$ & $\mathbf{1 . 4}$ & & 1.4 & 1.5 \\
\hline
\end{tabular}

CLR, context likelihood of relatedness; BN, bottleneck genes; NBN, nonbottleneck genes.

Differences in bold text are statistically significant $(p<0.05)$. 
Table 2. Shared Bottleneck Nodes between the ClR-Derived GSE2456 AND 15REgUlator NETWORKS

\begin{tabular}{|c|c|c|c|c|c|c|c|c|}
\hline$I D$ & $\begin{array}{l}\text { Gene } \\
\text { name }\end{array}$ & Description & $G C \%$ & $E C O$ & Mac & $M g M$ & VirSum & References \\
\hline STM2888 & spaR & $\begin{array}{l}\text { Surface presentation of antigens; secretory } \\
\text { proteins }\end{array}$ & $49 \%$ & $20 \%$ & 0.46 & 0.37 & 3 & $\begin{array}{l}\text { Chan et al., 2005; } \\
\text { Lawley et al., 2006; } \\
\text { Shah et al., } 2005\end{array}$ \\
\hline STM1404 & $\mathrm{sseF}$ & Secretion system effector & $49 \%$ & $0 \%$ & 3.37 & 3.15 & 2 & $\begin{array}{l}\text { Chan et al., 2005; } \\
\quad \text { Morgan et al., } 2004\end{array}$ \\
\hline STM1851 & & Putative cytoplasmic protein & $51 \%$ & $87 \%$ & 3.14 & 7.44 & 2 & $\begin{array}{l}\text { Chan et al., 2005; } \\
\text { Lawley et al., } 2006\end{array}$ \\
\hline STM2890 & spaP & $\begin{array}{l}\text { Surface presentation of antigens; secretory } \\
\text { proteins }\end{array}$ & $42 \%$ & $35 \%$ & 0.24 & 0.34 & 2 & Chan et al., 2005 \\
\hline STM3901 & ilvG & $\begin{array}{l}\text { Acetolactate synthase II, large subunit, } \\
\text { fragment } 1 \text {, cryptic }\end{array}$ & $56 \%$ & $47 \%$ & 0.79 & 0.84 & 2 & Chan et al., 2005 \\
\hline STM1540 & & Putative hydrolase & $48 \%$ & $0 \%$ & 2.11 & 1.22 & 1 & Shah et al., 2005 \\
\hline STM2139 & & Putative inner membrane protein & $51 \%$ & $\mathbf{0 \%}$ & 2.04 & 3.34 & 1 & Chan et al., 2005 \\
\hline STM2327 & nuoB & NADH dehydrogenase I chain B & $55 \%$ & $99 \%$ & 0.59 & 1.19 & 1 & Chan et al., 2005 \\
\hline STM2331 & yfbQ & $\begin{array}{l}\text { Putative aminotransferase (ortho), paral } \\
\text { putative regulator }\end{array}$ & $52 \%$ & $97 \%$ & 0.15 & 0.69 & 1 & Chan et al., 2005 \\
\hline STM2646 & yfiD & Putative formate acetyltransferase & $50 \%$ & $96 \%$ & 0.02 & 0.47 & 1 & Lawley et al., 2006 \\
\hline STM1367 & ydiH & Putative cytoplasmic protein & $46 \%$ & $80 \%$ & 0.45 & 0.81 & 0 & \\
\hline STM1369 & sufA & Putative HesB-like domain & $52 \%$ & $84 \%$ & 0.03 & 3.24 & 0 & \\
\hline STM1514 & ydeJ & Putative competence-damaged protein & $49 \%$ & $56 \%$ & 1.6 & 1.45 & 0 & \\
\hline STM1562 & & Putative periplasmic transport protein & $45 \%$ & $43 \%$ & 1.86 & 0.12 & 0 & \\
\hline STM1632 & & Putative inner membrane protein & $47 \%$ & $0 \%$ & 1.37 & 2.73 & 0 & \\
\hline STM1635 & & $\begin{array}{l}\text { Putative ABC-type polar amino acid } \\
\text { transport system }\end{array}$ & $44 \%$ & $39 \%$ & 5.57 & 3.42 & 0 & \\
\hline STM2148 & & Putative periplasmic protein & $48 \%$ & $82 \%$ & 1.47 & 1.20 & 0 & \\
\hline STM2325 & nuoE & NADH dehydrogenase I chain E & $56 \%$ & $97 \%$ & 0.35 & 0.92 & 0 & \\
\hline STM2892 & invJ & $\begin{array}{l}\text { Surface presentation of antigens; secretory } \\
\text { proteins }\end{array}$ & $49 \%$ & $0 \%$ & 0.13 & 0.43 & 0 & \\
\hline STM3069 & pgk & Phosphoglycerate kinase & $55 \%$ & $98 \%$ & 0.04 & 1.05 & 0 & \\
\hline STM3303 & rpmA & 50S ribosomal subunit protein L27 & $50 \%$ & $95 \%$ & 0.57 & 0.71 & 0 & \\
\hline STM3839 & $\mathrm{rpmH}$ & 50S ribosomal subunit protein L34 & $53 \%$ & $100 \%$ & 1.62 & 0.88 & 0 & \\
\hline STM4237 & lexA & $\begin{array}{l}\text { SOS response regulator, transcriptional } \\
\text { repressor (LexA family) }\end{array}$ & $55 \%$ & $97 \%$ & 8.98 & 0.91 & 0 & \\
\hline STM4363 & hflK & $\begin{array}{l}\text { Modulator for protease specific for FtsH } \\
\text { phage lambda cII repressor }\end{array}$ & $57 \%$ & $94 \%$ & 0.9 & 0.85 & 0 & \\
\hline
\end{tabular}

ECO, Escherichia coli homology (percentage protein sequence identitity); Mac, fold induction in macrophages at 8 h post-infection; $\mathrm{MgM}$, fold induction in low $\mathrm{Mg}^{2+}$ minimal media $(\mathrm{MgM})$ media; CLR, context likelihood of relatedness.

et al., 2007), we found a significant enrichment of essential genes in bottlenecks from networks inferred using CLR. Additionally, as reported in Yu et al. (2007), we found that the bottlenecks that are also hubs are even more enriched in essential genes. However, in one of our networks, the 15regulator network, the nonhub-bottleneck genes were also significantly enriched in essential genes, suggesting that bottlenecks have an important role in the network regardless of whether they are hubs. This is similar to results in experimentally derived regulatory networks (Yu et al., 2007), consistent with the intent of CLR to infer these networks (Faith et al., 2007). These results show that $30-40 \%$ of the essential genes in the network are bottleneck genes. Similar results were also obtained using the VirSum measure, indicating that bottlenecks are also essential under a greater range of conditions (mean VirSum 0.59) than non-bottlenecks (mean VirSum 0.28). Though we used a definition of bottleneck genes as genes with the top $20 \%$ of betweeness values as previously described (Yu et al., 2007), the enrichment of the essential genes in bottlenecks was also observed using the top $10 \%, 15 \%$, and $25 \%$ of betweeness scores.

The CLR algorithm calculates the mutual information (MI) from all pairs of expression patterns in a dataset, then applies a filter that assesses the statistical significance of each pair-wise MI score in the context of the local neighborhood of both genes involved to produce the final relationship zscores (Faith et al., 2007). We were interested in assessing the contribution from each part of this process to the observation that bottleneck genes were more likely to be essential in virulence than non-bottleneck genes. Accordingly, we used the raw mutual information values output from CLR (i.e., before statistical filtering of relationships) and PCC to generate networks from the GSE2456 dataset, and compared the enrichment of virulence essentiality in the resulting bottleneck genes (Fig. 1). We found that the bottleneck genes from 
Table 3. Functional Enrichment of Bottleneck Nodes

\begin{tabular}{lcc}
\hline & \multicolumn{2}{c}{ Fold enrichment $($ BN/NBN) } \\
\cline { 2 - 3 } \multicolumn{1}{c}{ Pathway } & GSE2456 & 15regulator \\
\hline Type III secretion system & $\mathbf{2 . 7 5}$ & $\mathbf{2 . 4 8}$ \\
Protein export & $\mathbf{1 2 . 6 8}$ & 0.00 \\
Fatty acid biosynthesis & $\mathbf{9 . 5 0}$ & 0.00 \\
Propanoate metabolism & $\mathbf{5 . 5 1}$ & 0.00 \\
Benzoate degradation via CoA ligation & $\mathbf{3 . 7 7}$ & 0.00 \\
Glycolysis/gluconeogenesis & $\mathbf{3 . 5 3}$ & 0.00 \\
Reductive carboxylate cycle & $\mathbf{3 . 5 3}$ & 0.00 \\
Pyruvate metabolism & $\mathbf{3 . 1 5}$ & 0.47 \\
Ubiquinone biosynthesis & $\mathbf{3 . 1 4}$ & 0.94 \\
Butanoate metabolism & $\mathbf{2 . 8 3}$ & 0.00 \\
Citrate cycle (TCA cycle) & $\mathbf{2 . 8 3}$ & 2.83 \\
Oxidative phosphorylation & $\mathbf{2 . 3 0}$ & 0.81 \\
Flagellar assembly & 0.00 & $\mathbf{3 1 . 1 8}$ \\
Phosphotransferase system & 3.14 & $\mathbf{8 . 5 0}$ \\
Ribosome & $\mathbf{0 . 2 6}$ & $\mathbf{2 . 4 6}$ \\
Any KEGG pathway & $\mathbf{1 . 3 1}$ & $\mathbf{0 . 7 2}$ \\
\hline Bold type indicates statistically significant enrichment $(p<0.05)$. & \\
BN, bottleneck genes; NBN, non-bottleneck genes; KEGG, Kyoto Encyclopedia \\
of Genes and Genomes. & &
\end{tabular}

these networks were not more likely to be essential in virulence, indicating that the filtering step in CLR is important for generation of biologically useful networks.

Genes essential in virulence show a greater degree of induction in macrophages and in culture conditions that mimic the intracellular macrophage environment ( $\mathrm{MgM}$ media) relative to non-essential genes (data not shown), though induction in macrophages does not necessarily demonstrate that a gene is essential for virulence or even involved in the virulence process. To assess the behavior of bottlenecks in virulence conditions, we compared the expression of bottleneck nodes and non-bottleneck nodes under these two conditions (Table 1). For macrophage expression, we used a global transcriptional profiling experiment on S. typhimurium isolated from host macrophages (Eriksson et al., 2003) at the 8- and 12-h post-infection time points. Culture of $S$. typhimurium in low $\mathrm{pH} / \mathrm{low} \mathrm{Mg}^{2+}$ minimal media $(\mathrm{MgM})$ has been found to mimic the intracellular environment and induce expression of a range of genes known to be involved in virulence. Transcriptional profiles from $S$. typhimurium grown in $\mathrm{MgM}$ were compared with expression in $\log$ phase growth in rich media to obtain the level of induction under infectious culture conditions. In both the GSE2456 and 15regulator CLR networks, bottlenecks were more induced in macrophages at $12 \mathrm{~h}$ post-infection, consistent with their demonstrated role in virulence. Also, in the 15regulator network, the level of induction in MgM media for bottlenecks was approximately three times that of non-bottlenecks. The 15regulator experiment used MgM media to induce virulence genes, suggesting that the behavior of bottleneck genes in inferred networks may be related to the conditions used to generate the gene profiles.

The bottlenecks and non-bottleneck genes were then compared using two different gene characteristics (Table 1). We found that the bottleneck genes from the 15regulator CLR network had a significantly lower $\mathrm{G}+\mathrm{C}$ content and were less well conserved in $E$. coli than their non-bottleneck counterparts. Both these tendencies are also observed in the total set of genes found to be essential in virulence (data not shown), further supporting the assertion that bottleneck proteins play a role in virulence.

We next investigated the set of bottleneck nodes that were shared by both CLR-derived networks. We used more generous thresholds for network generation (GSE2456 zscore of 6.3 and 15regulator zscore of 4.6) to provide a sufficient amount of overlap between the bottleneck genes to allow statistical analysis. This set of 24 genes is listed in Table 2. Comparing this list against the set of non-bottleneck genes combined from both networks inferred using the CLR algorithm showed that the shared bottlenecks were even more likely to be essential for virulence over a broader range of conditions (43\% VirEss, 0.65 mean 
VirSum). The list contains a number of genes known to be involved in virulence (invJ, spaP, spaR, and $s s e F)$. InvJ is a known virulence effector secreted by the type III system (Russmann et al., 2002). Like some other type III effectors, it is not essential for virulence, likely because it serves a redundant function (Galan et al., 1999). This suggests that other genes identified as bottlenecks, but not found on the lists of essential genes, might also be important in virulence. Importantly, the shared bottlenecks list contains three genes with unknown function that have been identified as being essential in virulence (STM1540, STM1851, STM2139), and these represent excellent targets for experimental investigation.

We were interested in examining the functions of the protein products of the bottleneck genes and so calculated the enrichment of functional categories obtained from the Kyoto Encyclopedia of Genes and Genomes (KEGG) (Kanehisa and Goto, 2000) in terms of fold-enrichment of the categories in the bottleneck group versus the non-bottleneck group in the two CLR-derived networks. Our first observation from this analysis was that the GSE2456 bottlenecks contained significantly more KEGG-annotated genes $(80 \%)$ than the bottlenecks from the 15regulator network (46\%) relative to the KEGG annotation of the non-bottlenecks ( $57 \%$ and $48 \%$, respectively). Table 3 shows the functional enrichment of categories with significant enrichment ( $p$-value $<0.05$ calculated using a chi-square test) in at least one of the two CLR networks. Type III secretion system components are enriched in both sets of bottlenecks, reflecting the importance of this system in virulence (Galan and Wolf-Watz, 2006). The GSE2456 bottlenecks are differentially enriched in a number of categories primarily in pathways involved in carbohydrate metabolism and energy metabolism. The 15regulator bottlenecks are differentially enriched in proteins involved in flagellar assembly and the phosphotransferase system. The implications of these findings are discussed below.

\section{CONCLUSION}

The findings presented here show that bottlenecks in inferred networks are more likely to be essential in virulence than their non-bottleneck counterparts. The percentage of virulence essential bottlenecks $(\sim 32 \%)$ compared to non-bottlenecks (19\%) in the two networks inferred using the CLR algorithm is comparable to the relationship observed between the essentiality of bottlenecks and non-bottlenecks in the yeast proteinprotein interaction network (34\% versus 19\%). The observed enrichment increased if the bottlenecks were limited to those that were also hubs (to $40 \%$ ), as previously observed, by considering bottlenecks that are shared between the two networks, and by using more conservative zscore thresholds for network generation. In the 15regulator CLR network a maximal significant enrichment is reached at 70\% (essential bottleneck genes) versus 30\% (essential non-bottleneck genes) for a limited number of genes (78 total). By contrast, bottleneck nodes in networks generated using simple mutual information and Pearson correlation were not more likely to be essential in virulence than their non-bottleneck counterparts. If bottleneck nodes are indeed a common feature of biological networks that have different properties than non-bottlenecks, as suggested by studies of several types of experimentally derived networks (Yu et al., 2007), this finding suggests that the CLR algorithm is identifying more biologically relevant relationships between genes than mutual information alone or Pearson correlation does. Interestingly, bottlenecks in the MI network are significantly depleted of genes essential in virulence (Fig. 1B) though the significance of this observation is unclear.

Bottlenecks also appear to be essential in a larger range of conditions, as judged by the VirSum measure. This is interesting, since the four studies from which the virulence essentiality list was compiled were for different cell types and hosts. Two studies used S. typhimurium SL1344: one assessed the impact of mutations on long-term survival in $129 \mathrm{X} 1 / \mathrm{Sv}$ mice 28 days post-infection (Lawley et al., 2006), and the other examined the impact of mutations on infection of cultured mouse RAW 264.7 macrophages at $12 \mathrm{~h}$ post-infection or BALB/cJ mice $48 \mathrm{~h}$ post-infection (Chan et al., 2005). Another study used S. typhimurium ST4/74 to infect calf or chick intestines and analyzed surviving bacteria at 3-5 days post-infection (Morgan et al., 2004). The final study used a related organism Salmonella gallinarum to infect chickens and analyzed 4 days post-infection (Shah et al., 2005). Addition of further studies of genes essential to the virulence of Salmonella is likely to improve the results presented here.

Bottleneck genes from our networks generated from experiments using in vitro culture conditions were significantly induced in macrophages at $12 \mathrm{~h}$ post-infection. This shows that the networks generated and 
topological analysis is correlated with virulence processes in vivo. Bottlenecks in the 15regulator network were more likely to be induced in $\mathrm{MgM}$ media. This observation suggests that the role of bottlenecks in inferred networks is related to the underlying experimental design, since the $\mathrm{MgM}$ media was a culture condition in the 15regulator dataset. This hypothesis is supported in the functional enrichment results discussed below.

We found that bottlenecks derived from both CLR-derived networks were significantly enriched in genes involved in type III secretion, which is not surprising given the central role of type III secretion in the virulence of S. typhimurium (Galan et al., 1999). Bottlenecks in the 15regulator CLR network were enriched in genes from the flagellar assembly and the phosphotransferase (PTS) systems. Both these pathways have a demonstrated role in virulence, flagella in cellular invasion (Parker and Guard-Petter, 2001), and PTS in metabolic regulation (Kok et al., 2003). Given this importance, the observed functional enrichment further suggests that bottlenecks play an important role in the virulence program. Bottlenecks from the GSE2456 network are enriched in genes involved in carbohydrate and energy metabolism. The GSE2456 dataset was a compilation of microarrays from bacteria grown under a number of different culture conditions, including multiple carbon sources (e.g., glucose, glycerol, acetate). This suggests that the bottleneck genes identified reflect the states of the system that are represented in the experimental design. One hypothesis is that bottleneck genes in inferred networks link modules that represent system states. This is supported by the observation that genes linking functional modules identified using hierarchical clustering in the GSE2456 network are predominantly bottlenecks (70\%; data not shown). The role of bottlenecks then may be as mediators of system state transitions by controlling transitions between distinct functional modules. An alternative, related hypothesis is that bottlenecks are sentinels of these systems transitions. Further computational and experimental investigation will be necessary to answer these questions.

The list of essential virulence genes compiled here covers a limited number of hosts/cell types and is almost certainly incomplete. Virulence-associated factors (such as invJ and other type III secretion system components and effectors), known to be involved in virulence but not found on the list of virulenceessential genes, are enriched in bottleneck nodes. These two considerations suggest that our findings represent a conservative estimate of the importance of bottleneck nodes in the virulence process. It is likely that experimental investigation of the targets identified here will elucidate more links with the virulence program in S. typhimurium.

We have presented findings showing that hubs and bottlenecks in networks inferred from two different global transcriptomic datasets using the CLR algorithm have characteristics distinguishing them from other genes in the networks. We demonstrated that bottleneck and hub genes were more likely to be essential in virulence and are essential in a broader range of conditions than non-bottlenecks. Further, we show that bottlenecks that are also hubs are more likely to be essential in virulence than non-hub bottlenecks. This is the first report of network topology being applied to inferred networks to determine essentiality. This approach has the potential to be broadly useful, and unlike high-throughput proteinprotein interaction data, large amounts of microarray data are available for a broad range of organisms under many conditions. Currently we are validating these findings using larger compendia of microarray datasets from S. typhimurium and using inferred networks in other pathogens. Given that protein-protein interaction networks can provide similar information it will be useful to develop better ways of merging these two types of networks. Our results suggest that this approach can reveal aspects of the underlying experimental design, and thus the biological principles represented by this design.

\section{ACKNOWLEDGMENTS}

The research described in this paper was conducted for the Biomolecular Systems Initiative under the Laboratory Directed Research and Development Program at the Pacific Northwest National Laboratory (PNNL), a multiprogram national laboratory operated by Battelle for the U.S. Department of Energy (under contract DE-AC06-76RL01830). In addition, SEBINI has been supported by PNNL's William R. Wiley Environmental Molecular Science Laboratory (EMSL) and the EMSL Grand Challenge in Membrane Biology project. 


\section{DISCLOSURE STATEMENT}

No competing financial interests exist.

\section{REFERENCES}

Adkins, J.N., Mottaz, H.M., Norbeck, A.D., et al. 2006. Analysis of the Salmonella typhimurium proteome through environmental response toward infectious conditions. Mol. Cell Proteomics 5, 1450-1461.

Altschul, S.F., Madden, T.L., Schaffer, A.A., et al. 1997. Gapped BLAST and PSI-BLAST: a new generation of protein database search programs. Nucleic Acids Res. 25, 3389-3402.

Angelichio, M.J., and Camilli, A. 2002. In vivo expression technology. Infect. Immun. 70, 6518-6523.

Chan, K., Kim, C.C., and Falkow, S. 2005. Microarray-based detection of Salmonella enterica serovar Typhimurium transposon mutants that cannot survive in macrophages and mice. Infect. Immun. 73, 5438-5449.

Darwin, A.J. 2005. Genome-wide screens to identify genes of human pathogenic Yersinia species that are expressed during host infection. Curr. Issues Mol. Biol. 7, 135-149.

Dyer, M.D., Murali, T.M., and Sobral, B.W. 2008. The landscape of human proteins interacting with viruses and other pathogens. PLoS Pathog. 4, e32.

Eriksson, S., Lucchini, S., Thompson, A., et al. 2003. Unravelling the biology of macrophage infection by gene expression profiling of intracellular Salmonella enterica. Mol. Microbiol. 47, 103-118.

Faith, J.J., Hayete, B., Thaden, J.T., et al. 2007. Large-scale mapping and validation of Escherichia coli transcriptional regulation from a compendium of expression profiles. PLoS Biol. 5, 54-66.

Ferrarini, L., Bertelli, L., Feala, J., et al. 2005. A more efficient search strategy for aging genes based on connectivity. Bioinformatics 21, 338-348.

Fields, P.I., Swanson, R.V., Haidaris, C.G., et al. 1986. Mutants of Salmonella typhimurium that cannot survive within the macrophage are avirulent. Proc. Natl. Acad. Sci. USA 83, 5189-5193.

Fraser, H.B., Hirsh, A.E., Steinmetz, L.M., et al. 2002. Evolutionary rate in the protein interaction network. Science 296, 750-752.

Galan, J.E., and Collmer, A. 1999. Type III secretion machines: bacterial devices for protein delivery into host cells. Science 284, 1322-1328.

Galan, J.E., and Wolf-Watz, H. 2006. Protein delivery into eukaryotic cells by type III secretion machines. Nature 444, 567-573.

Geddes, K., Worley, M., Niemann, G., et al. 2005. Identification of new secreted effectors in Salmonella enterica serovar Typhimurium. Infect. Immun. 73, 6260-6271.

Goni, J., Esteban, F.J., de Mendizabal, N.V., et al. 2008. A computational analysis of protein-protein interaction networks in neurodegenerative diseases. BMC Systems Biol. 2, 52.

Guerquin, M., McDermott, J., Frazier, Z., et al. 2009. The bioverse API and web application. In: Computational Systems Biology, McDermott, J., Ireton, R., Montgomery, K., et al., eds., Humana Press, Totawa, NJ (in press).

Horvath, S., and Dong, J. 2008. Geometric interpretation of gene coexpression network analysis. PLoS Comput. Biol. 4, e1000117.

Jeong, H., Mason, S.P., Barabasi, A.L., et al. 2001. Lethality and centrality in protein networks. Nature 411, 41-42.

Jones, B.D., and Falkow, S. 1996. Salmonellosis: host immune responses and bacterial virulence determinants. Annu. Rev. Immunol. 14, 533-561.

Kanehisa, M., and Goto, S. 2000. KEGG: Kyoto Encyclopedia of Genes and Genomes. Nucleic Acids Res. 28, 27-30.

Kok, M., Bron, G., Erni, B., et al. 2003. Effect of enzyme I of the bacterial phosphoenolpyruvate: sugar phosphotransferase system (PTS) on virulence in a murine model. Microbiology 149, 2645-2652.

Lawley, T.D., Chan, K., Thompson, L.J., et al. 2006. Genome-wide screen for Salmonella genes required for long-term systemic infection of the mouse. PLoS Pathog. 2, e11.

McDermott, J., Guerquin, M., Frazier, Z., et al. 2005. BIOVERSE: enhancements to the framework for structural, functional and contextual modeling of proteins and proteomes. Nucleic Acids Res. 33, W324-W325.

Milo, R., Itzkovitz, S., Kashtan, N., et al. 2004. Superfamilies of evolved and designed networks. Science 303, 15381542.

Milo, R., Shen-Orr, S., Itzkovitz, S., et al. 2002. Network motifs: simple building blocks of complex networks. Science 298, 824-827.

Morgan, E., Campbell, J.D., Rowe, S.C., et al. 2004. Identification of host-specific colonization factors of Salmonella enterica serovar Typhimurium. Mol. Microbiol. 54, 994-1010.

Ozgur, A., Vu, T., Erkan, G., et al. 2008. Identifying gene-disease associations using centrality on a literature mined gene-interaction network. Bioinformatics 24, i277-i285. 
Parker, C.T., and Guard-Petter, J. 2001. Contribution of flagella and invasion proteins to pathogenesis of Salmonella enterica serovar enteritidis in chicks. FEMS Microbiol. Lett. 204, 287-291.

Pletcher, S.D. 2004. Vital connections. Sci. Aging Knowledge Environ. 2004, pe19.

Porwollik, S., Frye, J., Florea, L.D., et al. 2003. A non-redundant microarray of genes for two related bacteria. Nucleic Acids Res. 31, 1869-1876.

Promislow, D.E. 2004. Protein networks, pleiotropy and the evolution of senescence. Proc. Biol. Sci. 271, 1225-1234.

Reiss, D.J., Baliga, N.S., and Bonneau, R. 2006. Integrated bi-clustering of heterogeneous genome-wide datasets for the inference of global regulatory networks. BMC Bioinform. 7, 1471-2105.

Russmann, H., Kubori, T., Sauer, J., et al. 2002. Molecular and functional analysis of the type III secretion signal of the Salmonella enterica InvJ protein. Mol. Microbiol. 46, 769-779.

Said, M.R., Begley, T.J., Oppenheim, A.V., et al. 2004. Global network analysis of phenotypic effects: protein networks and toxicity modulation in Saccharomyces cerevisiae. Proc. Natl. Acad. Sci. USA 101, 18006-18011.

Shah, D.H., Lee, M.J., Park, J.H., et al. 2005. Identification of Salmonella gallinarum virulence genes in a chicken infection model using PCR-based signature-tagged mutagenesis. Microbiology 151, 3957-3968.

Taylor, R.C., Shah, A., Treatman, C., et al. 2006. SEBINI: Software Environment for BIological Network Inference. Bioinformatics 21, 2706-2708.

von Mering, C., Krause, R., Snel, B., et al. 2002. Comparative assessment of large-scale data sets of protein-protein interactions. Nature 417, 399-403.

Worley, M.J., and Heffron, F. 2000. Identification of exported bacterial proteins via gene fusions to Yersinia pseudotuberculosis invasin. Methods Enzymol. 326, 97-104.

Yao, L., and Rzhetsky, A. 2008. Quantitative systems-level determinants of human genes targeted by successful drugs. Genome Res. 18, 206-213.

Yoon, H., McDermott, J.E., Porwollik, S., et al. 2009. Coordinated regulation of virulence during systematic infection of Salmonella enterica serovar typhimurium. PLoS Pathogens (in press).

Yu, H., Kim, P.M., Sprecher, E., et al. 2007. The importance of bottlenecks in protein networks: correlation with gene essentiality and expression dynamics. PLoS Comput. Biol. 3, e59.

Address reprint requests to:

Dr. Jason McDermott

902 Battelle Boulevard

P.O. Box 999, Mail Stop K7-90

Richland, WA 99352

E-mail: Jason.McDermott@pnl.gov 